\title{
Frequency response function shape differences as a sign of bricks elements destruction diagnostics
}

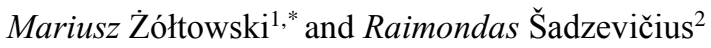 \\ ${ }^{1}$ W arsaw University of Life Sciences - SG GW, 02-787 N ow oursynowska 166, Poland. \\ ${ }^{2} \mathrm{~V}$ ytautas M agnus U niversity A griculture A cademy, $53361 \mathrm{~K}$ aunas, Lithuania.
}

\begin{abstract}
The knowledge of the dynamic state and structure of system allows describing its behavior and allows creating prognosis models of the system behavior in the function of dynamic evolution time, based on the model of the technical state symptoms growth. Most often, there are no known equations describing behaviors of the system in the function of dynamic evolution time, which accounts for the need to apply new tools to examine the dynamic state. In this article authors shows chosen problems of technical state diagnosis with the use of identification and technical diagnostics methods such as experimental modal analysis. Relations between methods of dynamic state evaluation and methods of technical state evaluation were indicated. Example modal analysis results illustrate the complexity of projecting dynamic state researches into diagnostic researches of state evaluation.
\end{abstract}

\section{Intorduction}

Destruction processes of technical systems force the need to supervise changes of their technical state. It is possible with the use of technical diagnostics methods.

Methods and means of modern technical diagnostics are a tool of machine state diagnosis, which is the basis of decisions made at each stage of their existence.

$M$ any previous works clearly indicate connections between dynamics and technical diagnostics, especially vibration diagnostics. The bases of identification, modeling and concluding fully convince towards the dominating role of vibrations in machine state identification [1]. Properly planned and realized experiment is the base to obtain diagnostically sensitive signals, which processed will determine state diagnosis procedures. The processing includes creation of numerous signal measures in time domain, frequencies and amplitudes, selection and reduction of the number of signal measures, creation and analysis of effectiveness of cause-and-effect models, as well as evaluation of the righteousness of made diagnostic decisions [2].

\section{Dynamics and diagnostics}

Into quality measures of machine's technical state, i.e. its dynamics, included is the level of vibration amplitudes, as well of the machines as the lot, and also of relative vibrations of separate elements and parts. Overall vibrations of the machine can be perceived as an external symptom while they are responsible for the level of interferences emitted into the environment. Relative vibrations of separate elements, however, influence mainly the state of internal forces in the machine, i.e. at its level of dynamic stress amplitudes [3].

Identification can concern both the construction of models and the reconstruction of the examined model state, which leads straight to the problem of technical diagnostics.

* Corresponding author: zoltowskimariusz1981@gmail.com 
The process of diagnostic identification includes modeling (symptom or structural), identification experiment (simulation and/or real), estimation of diagnostic parameters (state features or symptoms), diagnostic concluding. The specificity of diagnostic identification tasks is different from general identification in the way that it includes a number of additional elements enhancing this process. They are:

- constructing models of diagnostic signals generation,

- choosing features of object structure state and diagnostic symptoms,

- modeling cause-and-effect relations,

- evaluating the accuracy of choosing variables for the model,

- determining boundary values of the symptoms,

- state classification and determining of periodicity a diagnosis.

M ethods of identification can be divided concerning: the kind of identified model, the kind of experiment, identification criterion applied, as well as estimation procedure applied. In general, these are: methods of analysis, time, frequency, correlation, regression, factor analysis, as well as iteration methods described in works of many authors [4].

For simple objects a good tool to evaluate their changeable dynamic state are methods of simple identification, which use amplitude - frequency spectrum. Searching resonance frequency and amplitude value in this frequency with the use of tests (impulse, harmonic and random) are relatively well mastered in research techniques of our enterprises [5].

A nother way of describing and analyzing the dynamic state of machines is a modal analysis used as a theoretical, experimental and exploitation method. It uses frequencies of own vibrations, values of suppression and forms of vibrations to describe the changing machine state, and it is used to improve the finished elements method. The presented procedures are based on the knowledge of the system model, and the conclusions drawn from the actions on the models depend on their quality. Depending on the aim of the performed dynamic analysis of the object, different requirements are set for the constructed models, and their evaluation is conducted with different experimental methods.

\section{Description of object state changes}

The dynamic state of the object can be, in the easiest case, described with a model of 1 degree of freedom - Fig.1. A conventional description of this model is known relations indicating that vibrations well reflect the state of the machine. A description of this model can be achieved within $m, k, c$ categories, or through $a, v, x$ researches [6].
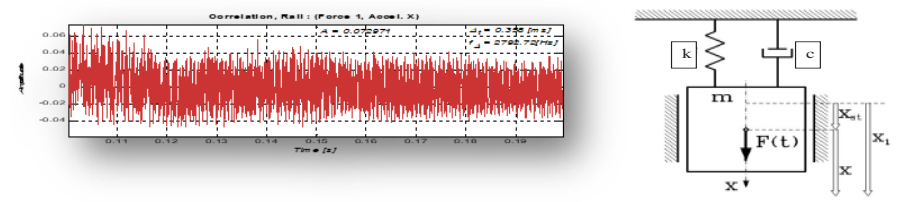

Fig.1. System model of 1 degree of freedom and vibration signal of real system.

"the state parameters $(m, k, c)=$ vibration process $(a, v, x)$ "

$$
\begin{gathered}
m \cdot \ddot{x}+c \cdot \dot{x}+k \cdot x=F(t) \\
x=A \sin (\varpi \cdot t+\phi) \\
v=\frac{d x}{d t}=A \varpi \cos (\varpi t+\varphi) \\
a=\frac{d^{2} x}{d t^{2}}=\frac{d V}{d t}=-A \varpi^{2} \sin (\varpi t+\varphi)
\end{gathered}
$$


Identification of his model (Eq.1) from the experimental side is the $a, v, x$ measurements for different time moments, which reflects the changes of the object state and is widely applied in vibration diagnostics. The solution of the task in the $m, k, c$, categories, however, requires a number of solution conversion of the equation (Eq. 1) for determining:

$$
\begin{gathered}
c_{k r}=2 m \varpi \quad c_{k r}=4 \Pi m f \\
k=m \cdot \varpi^{2} \quad k=4 \Pi^{2} m f^{2}
\end{gathered}
$$

Determining the value (5) requires realizing identification experiment from which the frequency $f$ or frequency $\omega$ can be determined. Here is useful the simple identification or modal analysis directly giving the values of own frequencies $\omega$ from the stabilization diagram - Fig. 2.

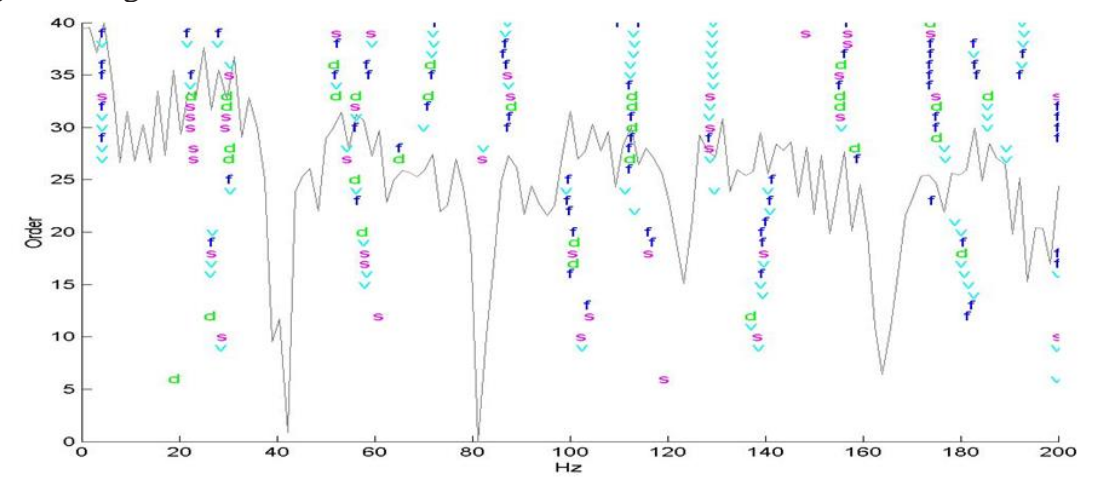

Fig.2. Stabilization diagram for $\omega$ determination.

The problem becomes more complicated for models of many degrees of freedom (more than 3). Here also the problem of object state identification can be solved from the measurement side $(a, v, x)$, Chile from the side of determining $\mathrm{m}, \mathrm{k}, \mathrm{c}$ own problem needs to be solved [7].

$$
\left(K-\omega^{2} M\right) \cdot q_{0}=0
$$

Equation (Eq. 3) presents a linear system of homogeneous al gebraic equations:

$$
\begin{gathered}
\left(k_{11}-\omega^{2} m_{11}\right) q_{1}+\left(k_{12}-\omega^{2} m_{12}\right) q_{2}+\cdots+\left(k_{1 n}-\omega^{2} m_{1 n}\right) q_{n}=0 \\
\left(k_{21}-\omega^{2} m_{21}\right) q_{1}+\left(k_{22}-\omega^{2} m_{22}\right) q_{2}+\cdots+\left(k_{2 n}-\omega^{2} m_{2 n}\right) q_{n}=0 \\
\cdots \cdots . . . \cdots \\
\left(k_{41}-\omega^{2} m_{41}\right) q_{1}+\left(k_{42}-\omega^{2} m_{42}\right) q_{2}+\cdots+\left(k_{n n}-\omega^{2} m_{n n}\right) q_{n}=0
\end{gathered}
$$

A solution for $q \neq 0$ exists when the main matrix determinant $\left(K-\omega^{2} M\right)=0$, i.e. $\operatorname{det}\left(K-\omega^{2} M\right)=0$. Solving the system of Eq. 4 own values can be determined, and from them the frequencies of own vibrations, indispensable for the object identification.

$$
\left(\lambda=\omega^{2}=\frac{k}{m}\right)
$$

\subsection{Environment of experimental modal analysis}

For complex systems, nonlinear often used for identifying complex modern method of noninvasive test, which is the modal analysis. As a result of modal analysis is obtained modal 
model, which is an ordered set of its own frequency, the corresponding damping ratios and mode shapes. Based on knowledge of the modal model of the object can be predicted response to any disorder in both time and frequency domains [8].

Using the statement that the destruction of the state of the object (test material) can be described alternatively [instead of modelling the changes in terms of the $(m, k, c)$ use the description of the vibration in terms of $(a, v, x)]$ in the study of the development of the state of degradation of the structure or masonry vibration frequencies were evaluated, resulting directly from the application of modal analysis procedures [9].

The identification of the individual modal models for extortion in the test sub-element and further aggregating them for the whole structure of the object is obtained evolutionary model, clearly describing the changes estimators state variable load conditions. Is revealed by the fractal nature of energy conversion the processes, and perfecting possible ways to zoom description of the real world. In most practical applications require the analysis of a multi-modal experiment and the complex calculations associated with the processing of the measured signals and model parameter estimation. So, welcome the possibility of applications allows us to distinguish the following types of modal analysis:

- theoretical MA, which requires the solution of the problem for the assumed its structural model of the object,

- experimental MA, controlled experiment requiring identification, during which forces the movement of an object (eg. Vibrations) and measures the force and measure the responses in a number of measuring points distributed over the examined object,

- operational (exploitation) MA, based on a real experiment, in which measurements are made only in response to the many points of measurement, while the movement of the subject is due to the actual operating extortion.

The theoretical modal analysis is defined as the problem of self-dependent mass matrix, rigidity and damping. The theoretical modal analysis requires addressing issues of their own for the assumed of structural model. Here designated frequency sets its own frequency attenuation coefficients for their own forms of vibrations and allow the simulation of structural behaviour at any extortion, selection of controls, and other design modifications. It is used in the design process, when there is no possibility of carrying out studies on the subject. Modal analysis is widely applied resulting damages removing it from vibrations, modifying structure dynamics, updating analytical model or state control, and also used for monitoring vibrations in aircraft industry and civil engineering [9].

Theoretical modal analysis is defined as a matrix eigenvalue problem of dependent matrices of mass, stiffness and damping. It requires the eigenvalue problems for an A ssumed structural model of investigated structure to be solved. The determined sets of natural frequencies, damping coefficients for the natural frequencies and forms of natural vibrations make it possible simulate behaviour of a structure under arbitrary excitations, choice of steering means, modifications and other structural issues.

A nalysis of natural frequencies and vectors is obtained on the basis of motion equations (after neglecting terms which contain external damping matrix and load vector). Then the motion equation of natural vibrations helps us obtains the form:

$$
B \ddot{q}+K q 0
$$

For one d.o.f. system its solution is as follows:

$$
\phi(t)=\vec{\Phi} \text { i }(\phi)
$$

where:

- vector $\vec{q}$ of amplitudes of natural vibrations. 
On substitution of the above given equation and $2^{\text {nd }}$ derivative to the motion equation the following is obtained:

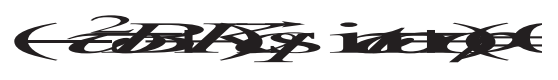

The equation is to be satisfied for arbitrary instant $t$, then the set of al gebraic equations is yielded as follows [14,19]:

$$
(K \underset{2}{\vec{a}}=\mathrm{C}
$$

This way was produced the set of linear homogeneous algebraic equations, which has non-zero solution only when the condition is fulfilled:

\section{$\operatorname{det}\left(\cos ^{2}\right)=c$}

On transformations the n-order polynomial is obtained. A mong its roots multifold ones may be present, and the vector built from the set of frequencies $\varpi^{2}$ ordered according to increasing value sequence is called the frequency vector, and the first frequency is called the fundamental one [11]:

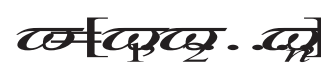

\subsection{Experiment in modal analysis}

Experiment to identify the state of the tested masonry destruction is the primary source information and on the basis of measurement values can be determined and the structure of the model. On the one hand, the quality of the experimental results obtained depends on the quality of the model, on the other hand the way the experiment determines the structure of the identified model [7].

The experiment of modal analysis can be divided into the following steps:

1) Planning the experiment:

- the choice of how to force vibration test piece and the points of application,

- selection of measurement points and vibration measuring equipment,

- selection of appropriate measuring equipment,

- selection of the model (reducing the number of degrees of freedom) of the system.

2) Calibration of the measurement path.

3) Acquisition and processing of the results of the experiment.

The aim of the experiment is to force modal analysis of the movement of the test piece masonry by providing energy and measuring the answer to extortion. The general procedure for carrying out studies of this work is shown in Figure 3.

MODAL TEST

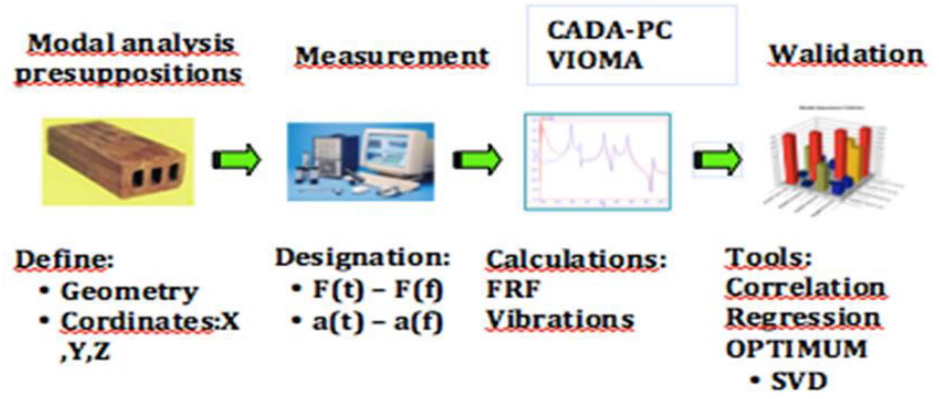

Fig.3. The essence of the measurement channel using modal analysis. 
The test piece subjected to forcing the forced walled corresponds to the vibration signal, proportional to the state of destruction. Forcing and response signal is used to determine the function of further FRF and the stabilization of the diagram, and the frequency of oscillations. By the way, these procedures are available for other interesting cognitive processes vibration estimators, which are also used in further studies. Test results after processing by different algorithms are subjected to statistical study. From the viewpoint of experimental modal analysis methods can be divided into:

- a method of forcing movement of the multiple actuators for the excitation of one form of vibration;

- the method of forcing movement of the one or more points in order to measure the transfer function.

The first group of methods is carried out manually moving the system in such a way as to force the vibration in accordance with one embodiment of the vibration. This requires a complex control system actuator to obtain the appropriate phase shifts force. The second group is used to force any depending on the type of the object. Set of equipment for the implementation of the experiment modal analysis is made up of components:

- measurement of the force of motion and response measurement

- signal conditioning system (pre-processing),

- the processing and collection of signals,

- forcing signal generation system,

- the vibration excitation.

The simplest due to maintenance solution is to use signal analyser, but the most modern giving the greatest potential solution is based on a workstation, and a specialized interface measurement. The basic operation performed by all applicable devices measuring modal analysis of signal to digital processing, which enables the use of digital signal processing techniques to determine the modal analysis required by the characteristics of estimators.

M odal studies do not care that the size of the kinematic motion measuring, as a response of the system. In practice, the displacement measurements give better results in terms of low frequency, and acceleration of the high frequency range. It is widely believed that the velocity measurements are the most optimal structural dynamics studies due to the fact that the effective value of the vibration velocity is in some sense a measure of the kinetic energy of vibration of the system. However, sensors for measuring displacement and velocity are relatively heavy and may affect the behaviour of the test object and the acceleration sensors have a considerably lower mass and therefore do not affect the motion of the system. An additional advantage of the acceleration sensor is that the acceleration signal a further signal can be obtained by integrating the velocity or displacement vibrations. Operation in the opposite direction, which consists of differentiation, can lead to large errors, especially in the higher frequencies.

On those grounds, acceleration sensors are the most commonly used transducers for studies of modal structure. A cceleration sensors built on the piezo-electric phenomena can be modelled as a system with one degree of freedom from suppression. The weight of this model is the seismic mass aggravating crystal piezoelectric material during movement. Due to the design of the sensors has its resonance, which reduces the frequency range in which they can be applied.

A very important factor influencing the modal test results associated with a variety of sensors, sensor-mounting location. The sensors should be mounted in such a way as not to affect the vibration system and are secured at the characteristic behaviour of the structure. Currently, the vibration measurement during the examination of the structure modal contactless sensors, are used and one of the feasibility of this type of sensor is the use of a laser beam. Such sensors enable the measurement of vibration velocity in the frequency range of 0 to $50 \mathrm{kHz}$, and amplitudes in the range of 0 to $100 \mathrm{~mm} / \mathrm{s}$. A n important factor in 
the range of measured frequency of the sensor is no way for the study of the structure. The sensors can be attached to the test structure by means of a special wax, adhesive, magnet or screw with the screw.

Experimental modal analysis requires laboratory conditions for testing. M odel is subject to advance well-known and established extortion. Forcing these may differ from those that operate on the object during normal operation. During the execution of the experiment may encounter difficulty in keeping in line with the reality of such boundary conditions, for example method of fixing the test object. In the case of large models, the execution of the experiment is very expensive and often impossible.

\section{Measurement software, and results}

For the measurement waveforms extortion and response system and determine the most used functions FRF measurement equipment purchased for the project company under the name of LMS TEST.XPRESS. This software enables you to easily perform a modal analysis of brick elements, as well as any other building structures. For the purposes of measurement using experimental modal analysis you defined two measurement channels. According to the theoretical experimental modal analysis first sensor is reserved for the modal hammer (vibration force), and second piezoelectric sensor is connected on the wall element (the answer key to force).

The next step was to prepare the masonry. In the study, it was decided to check is it possible to see a difference in destruction state in bricks, with use of only FRF function. For this purpose, were use 2 types of the samples. First sample was a full brick obtained from the brickyard, and for comparison a cracked (seen cracks on surface) full brick were also measured. For a better visualization of the results of the investigation, the results are shown below separately - 5 times is shown the FRF function for good, and 5 times for destroyed brick element. In Figure 4 it is shown once the extortion, and the answer of signal in time domain, which allows gaining $F R F$ function.

FRF - (Frequency Response Function) can be described as a quotient of the Fourier transform vibration exciting force $F(\omega)$, the Fourier transform of the response signal $A(\omega)$.

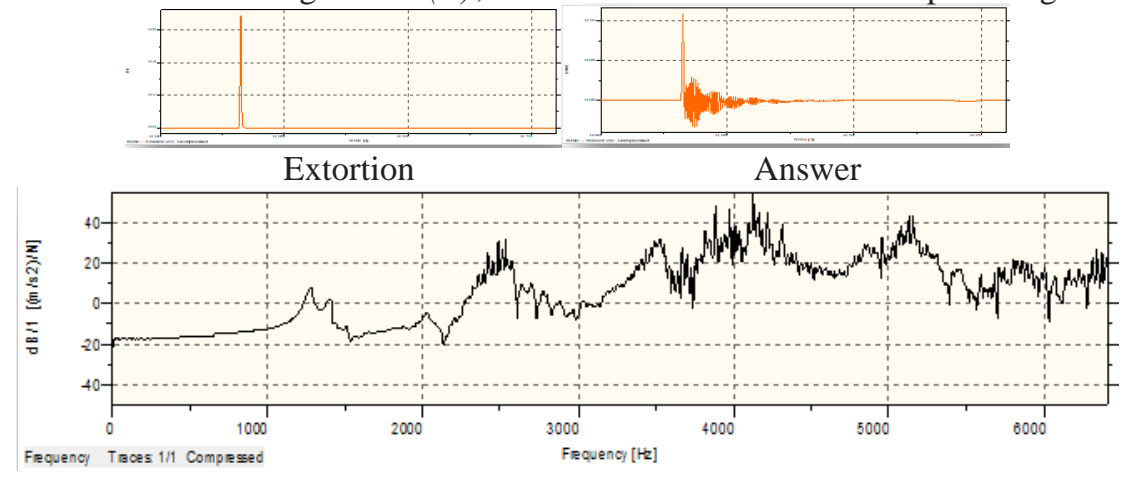

FRF

Fig. 4. Composition of results of measurements (the temporary course of extortion, temporary course of answer, function the FRF) the full brick in axis X.

During the tests it was able to generate a transfer functions of vibration signal by the structure (FRF function). The results are presented in real time in the center of a screen. It's allows visualizing the temporary courses of extortions and the answer, as well as the function the FRF and the function of coherence.

Graphic results, which show FRF functions of good, and destroyed bricks measured in axel $Y$ and 6 measurements for each material sample are show below in figure 5. 

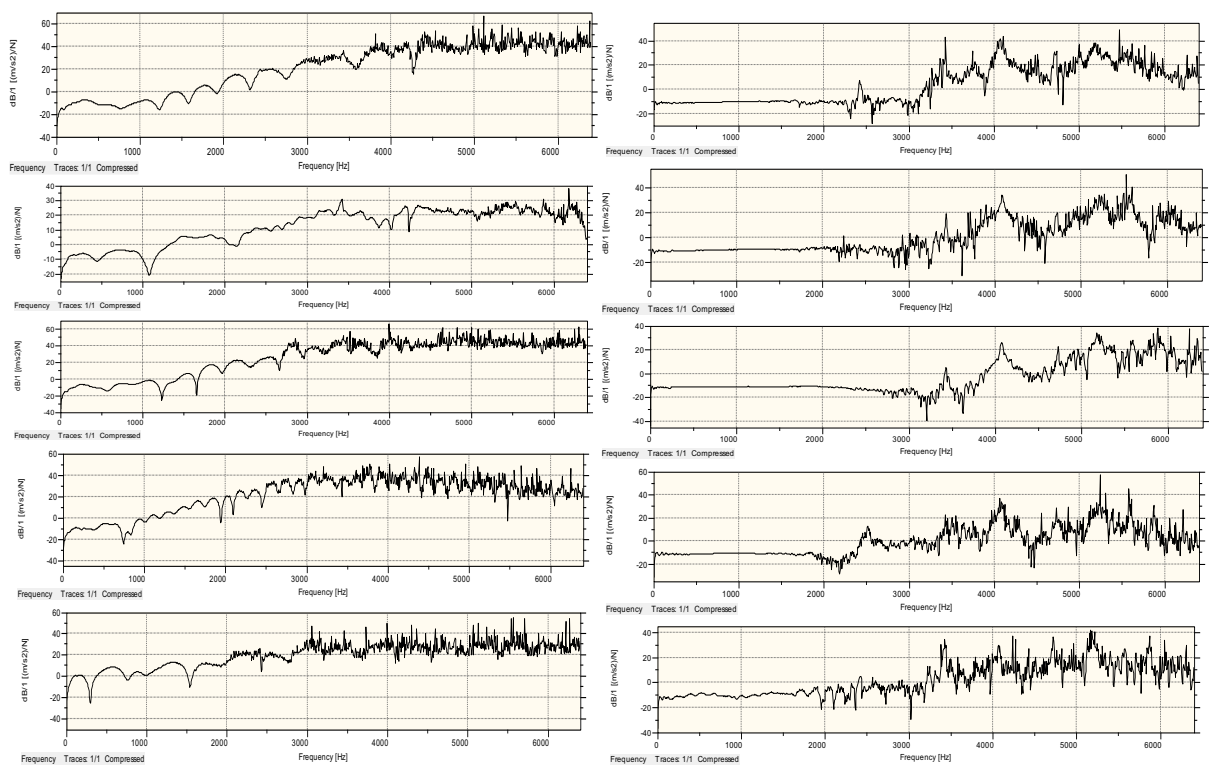

Fig. 5.Composition of FRF functions of full brick (good -left, damaged - right) in axis $Y$.

The results clearly differentiate the state of bricks degradation and in further research to indicate the usefulness of FRF be determined limit states in industrial diagnostic research.

\section{Summary}

Sulfur concrete is prepared hot by mixing modified sulfur cement and additives. M ethods of identification in the research building construction (including construction materials) are utility methods to estimate changes in operating structure. Civil engineers are increasingly using modal analysis of the varieties for its realization and modal model accurately reflects the destruction of objects.

Searching for mapping models with models of modal vibration, bench research and studies on real objects, allows assessing the similarity of the models, the relevance and effectiveness of decision methods. The search for methods of non-destructive testing of materials and structures indicates the possibility of using modal analysis in the assessment of their degradation, as shown in this study.

Practically verified susceptibility modal analysis of the degree of degradation of masonry shows to the satisfaction of practice differences between of the structure can bear and damaged. It is therefore possible to determine the risks of building structures on the basis of the study of natural frequencies and their characters, using operational modal analysis.

The results point to the fact that it is possible to distinguish between material properties, which has an impact on the ability to distinguish between their mechanical properties. The study also confirmed the usefulness of the LMS test apparatus using operational modal analysis performed on the actual building construction.

By obtaining graphical charts of FRF function, and a later their comparison it is possible to observe their diversity. These charts are different for materials that are in good condition, and damaged, which demonstrates the ability to assessment of the destruction of a brick element. 
It practically verified the sensitivity of assessment of modal analysis to degree of brick structure degradation. It becomes possible to determine hazards to a building structure on the basis of examining values of frequencies.

\section{References}

[1] B. Żółtowski, M. Żółtowski, Vibration signals in mechanical engineering and construction, (ITE-PIB 2015)

[2] B. Żółtowski, C. Cempel, Engineering of diagnostics machines, (PTDT, ITE - PIB 2004)

[3] M. Żółtowski, B. Żółtowski, Vibrations signal to the description of structural damage of dynamic the technical systems, (XIII International Technical Systems Degradation Conference 2015)

[4] M . Żółtowski, Opis drganiowy konstrukcji budowlanych, Logistyka nr.6/2014 (2014)

[5] M. Żółtowski, Investigations of harbor brick structures by using operational modal analysis. Polish M aritime Research, N 0. 1/(81), vol. 21 (2014)

[6] M. Żółtowski, M. Liss, The use of modal analysis in the evaluation of welded steel structures, Studies and Proceedings of Polish Association for Knowledge M anagement, V ol. 79 (2016)

[7] M. Żółtowski, M. Liss, Zastosowanie eksperymentalnej analizy modalnej w ocenie zmian sztywności prostego elementu konstrukcyjnego, Studies and Proceedings of Polish Association for K nowledge M anagement, V ol. 80 (2016)

[8] M. Żółtowski, R.M. M artinod, Technical Condition Assessment of Masonry Structural Components using Frequency Response Function (FRF), M asonry International J ournal of the International M asonry Society, V ol. 29, N 0.1, (2016)

[9] M. Żółtowski, R.M. Martinod, Quality identification methodology applied to wallelements based on modal analysis, Civil Engineering the Athens Institute for Education and Research, Emerald, A thens (2015) 